\section{Implications of the equivalence of mirror- image stimuli for object constancy}

ERNEST A. LUMSDEN, JR., University of North Carolina at Greensboro, Greensboro, N.C. 27412

The finding of mirror-image equivalence of two-dimensional stimuli is interpreted as a misapplication of processes normally contributing to object constancy. The generalized effect of reinforced responding to only one orientation of a three-dimensional object is reported for four pigeons and one rhesus macaque monkey. It is suggested that the mirror-image equivalence that is evidenced in these data with a three-dimensional object, plus the generalization from the mirror-image, as well as the reinforced orientation, may well provide the basis for data traditionally interpreted as "object constancy."

It is a general finding that animals and children require considerably more trials to discriminate between two identical forms differing only in orientation when the forms are oriented to constitute lateral mirror images of each other. Forms standing in this relationship (frequently referred to as an $\mathrm{H}$-reversal) are more difficult to discriminate than any other spatial relationship of identical forms, including a vertical mirror-image transformation (V-reversal). This has been found to be the case with octopuses (Sutherland, 1957), rats (Lashley, 1938), cats (Warren, 1969), rhesus monkeys (Riopelle et al, 1964), and children (Rudel \& Teuber, 1963). Both Lashley and Riopelle account for this finding by pointing to independent evidence that their Ss primarily attend the lateral edges of stimuli, especially the bottom edge, and discriminate between patterns on the bases of differences occurring in that region. Inasmuch as the lateral edges of H-reversals are much more similar than are the lateral edges of two identical forms in any other juxtaposition, it necessarily follows that two such forms should be the least discriminable. Sutherland (1957), on the other hand, proposes a scanning mechanism that operates in a vertical direction that is especially sensitive to the horizontal extent of the stimulus being scanned. For such a mechanism, the most readily discriminable orientations of two

Fig. 1. The projective geometry of the $S+$ object at orientations differing by $22.5 \mathrm{deg}$.
Other white three-dimensional object forms of the same height and volume were utilized as S-s. A CRF schedule of reinforcement was gradually reduced to a VI $30 \mathrm{~min}$ during the discrimination training of the first $S$ - from the $S+$. Training continued with this $S-$ alone until $80 \%$ of all responses for a daily session (45 $30-\mathrm{sec}$ stimulus presentations) were to the S+ object. At that point, another $S$ - object was introduced and the animal trained to criterion, and so on, until $S$ failed to respond initially to the presentation of a new S- (a novel stimulus). This state of affairs was deemed necessary before one could justifiably infer stimulus equivalence if, upon testing, $S$ responded to novel orientations of the $\mathbf{S +}$ object at a rate comparable to the rate emitted to the training orientation. For the pigeons, this state of "S+ conditioning" required five $S-s^{3}$; for the rhesus, four $S-s$ were required.

\section{TESTING}

A warm-up period was utilized consisting of three reinforced presentations of the 45-deg orientation of the S+ object. Then randomized blocks of presentations of the S+ object at test orientations of 0 , $45,90,135$ (mirror-image orientation of the 45 -deg orientation), and $180 \mathrm{deg}$ were interspersed among presentations of the $S-$ objects and several reinforced presentations of the S+ object at $45 \mathrm{deg}$. Of course, responding to the $S+$ object was never reinforced for any of the test orientations. RESULTS

Figure 2 contains the generalization gradients for each pigeon averaged across five such tests per $S$, as well as the gradient for the one rhesus averaged across two such tests. The slope of the individual daily gradients (of which each of these is the average) became steeper with increased testing. One need not subscribe to the "inverse hypothesis" that an organism generalizes to the extent that he fails to discriminate in order to relate these averaged generalization gradients to the discrimination data referred to in the introduction. When one recognizes that discrimination training is intrinsic in generalization testing in which responding is maintained by aperiodic St periods, these gradients can be viewed simply as reflecting the average level of
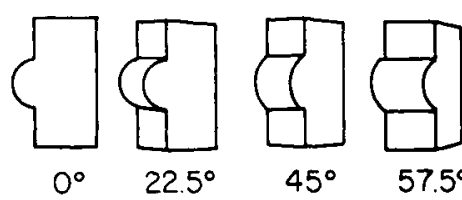

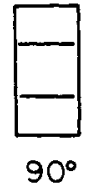

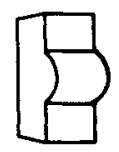

$112.5^{\circ}$

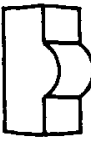

$135^{\circ}$

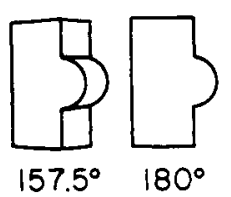

ORIENTATION 
Fig. 2. Relative response rate to test orientations of the $S+$ object (generalization gradient) for four pigeons and one rhesus macaque.

discrimination attained in a multiple-discrimination task in which the 45-deg orientation was only aperiodically reinforced. It is clear that for the pigeons the mirror-image (135-deg) orientation is more nearly equivalent to the S+ $(45-\mathrm{deg})$ orientation than is the $90-\mathrm{deg}$ orientation. For the rhesus, the mirror-image orientation is more nearly equivalent to the $\mathrm{S}+$ orientation than either the $90-$ or $0-\mathrm{deg}$ orientation, though the mirror image is less equivalent in an absolute sense than it is for most of the pigeon Ss.

DISCLISSION AND CONCLUSION

Elaborating upon a point made in the introduction, consecutive orientations of a solid object differing by 1 deg project 360 different planimetric geometric patterns. It now seems that conditioning to one of these projections results in considerable response strength to the mirror-image projection as well. So, disregarding the possibility of generalized response strength from the training stimulus and from the mirror image of that stimulus, the organism would have to be conditioned to 180 of these projections in order to respond appropriately to 360 orientations of that three-dimensional solid. The basis for natural selection of mirror-image equivalence is clear only when one considers that in the natural environment mirror-image patterns are projected only from the same object at differing orientations. (The orientation of a three-dimensional object that will project a retinal pattern that is the lateral mirror image of a particular orientation is that orientation differing from frontal parallel by the same number of degrees in the opposite direction.) From this point of view, the finding of mirror-image equivalence of two-dimensional (planimetric) stimuli can be considered as a misapplication of processes normally contributing to shape constancy in the same sense that Gregory (1966) considers

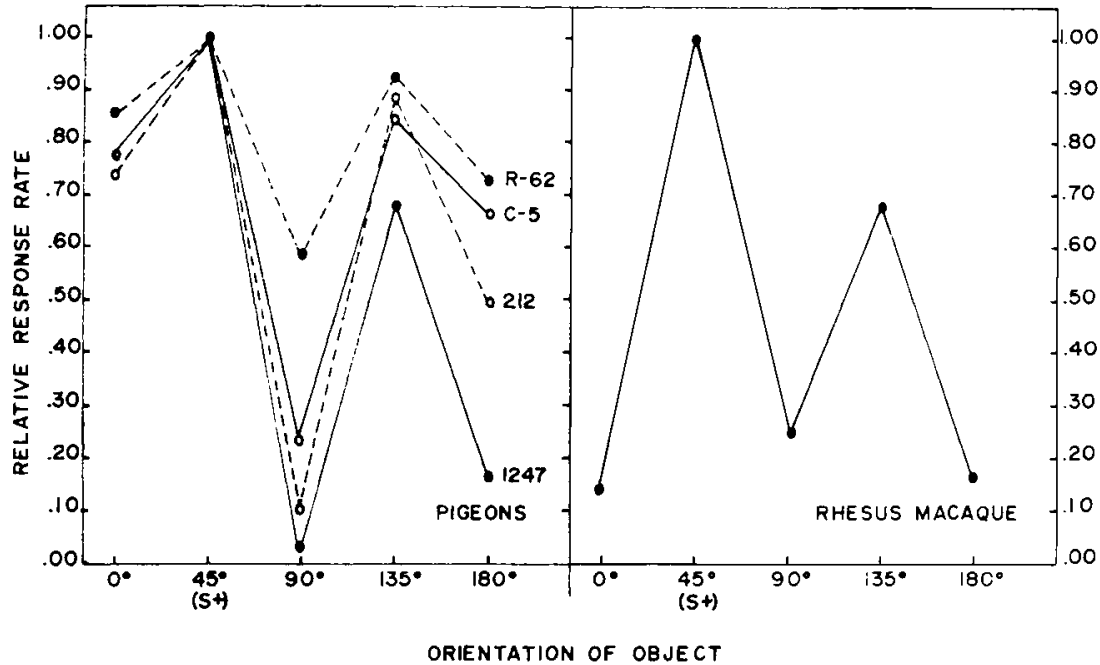

the Müller-Lyer illusion as resulting from a misapplication of processes normally contributing to size constancy.

The author has pilot data with pigeons that indicate that orientations intermediate to those tested here (i.e., 22.5, 57.5, 112.5 , and $157.5 \mathrm{deg}$ in Fig. 1) are responded to at rates very close to what one would interpolate from the data of adjacent orientations. Thus, it seems that the perceptual advantage afforded the organism by mirror-image equivalence is enhanced by generalization effects around the mirror-image orientation as well as around the reinforced orientation: Thus, certainly less than 180 orientations have to be reinforced before an animal responds to an object in such a way as to provide a basis for traditionally inferring object constancy. Guttman (personal communication), the author's mentor, has suggested that object constancy might be a complex instance of stimulus generalization. The ramifications of this provocative and parsimonious hypothesis are now being tested at this laboratory.

\section{REFERENCES}

GREGORY, R. L. Eye and brain, the psychology of seeing. New York: McGraw-Hill, 1966.

LASHLEY, $K$. S. The mechanism of vision: XV.
Preliminary studies of the rat's capacity for detail vision. Joumal of Genetic Psychology. $1938,18,123-193$

DRAPER, W. A. Discrimination of mirror-images by rhesus monkeys. Perceptual \& Motor Skills, 1964, 19, 383-389.

RUDEL, R. G., \& TEUBER, H. L. Discrimination of direction of line in children. Journal of Comparative \& Physiological Psychology. 1963, 56, 892-898.

SUTHERLAND, N. S. Visual discrimination of the orientation of rectangles by Octopus vulgaris Lamarck. Journal of Comparative \& Physiological Psychology, 1957, 48, 55-71.

WARREN, J. M. Discrimination of mirror im ages by cats. Journal of Comparative \& Physiological Psychology. 1969. 69, 9-11.

\section{NOTES}

1. The investigation with pigeons was conducted in 1961, while the author was in graduate training at Duke Ĺniversity under the direction of Dr. Norman Guttman. Dr. Guttman originated the idea of investigating object identification utilizing the generalization paradigm.

2. The investigation with rhesus macaques was sponsored by NSF RPTCT at the University of Michigan with Dr. Charles Butter. Other Ss and other variables were involved but are not pertinent to this presentation.

3. A more systematic investigation is now under way of the number of $S-S$ required for $S$ to respond to a novel stimulus on the basis of a comparison of its attributes with the reinforced stimulus rather than with the attributes of previously discriminated $\mathrm{S}-\mathrm{S}$.
RIOPELLE, A. J., ITOIGAWA. N.. RAHM. U.. \& 